\title{
تطوير الوسيلة التعليمية بنظام الإنجليزية بدون صعوبة باستخدام تطبيق أندرويد لمهارة الكلام
}

\author{
Robby Arinil Asror \\ e-mail: robby.arinil@gmail.com \\ Institut Agama Islam Negeri Kediri \\ Halil Thahir \\ e-mail: halilthahir16@yahoo.com \\ Institut Agama Islam Negeri Kediri

\section{Mokhammad Miftakhul Huda} \\ e-mail: hoeda_twin@yahoo.com \\ Institut Agama Islam Negeri Kediri
}

ملخص: أهداف هذا البحث لإنتاج الوسيلة التعليمية بنظام الإنجليزية بدون صعوبـة باستخدام تطبيق أندرويـد لترقية مهارة الكلام فى اللغة العربية، ولمعرفة تصحيح المنتج و لمعرفة نموذح التعلم المناسب لتطبيق المنتج. ويستخدم هذا البحث بنموذج التطوير من ديك وكاري. المنتج المطور هو تطبيق أندرويد يسمى ب"سهلة"، فياه ثلاثة موضيوعات وهي: الأسرة، و التعارف، و بيتي. و لكل موضوع أربعة مكونات على الأقل، منها النص و الفسديو و القصية القصيرة والمفردات. نتيججة تصحيح المنتج تدل على أن النتيجة من خبير المادة هي ه. ــ\% في فئة جيدة و النتيجة من خبير الوسيلة هي ا. 1.17 في فئة جيدة أيضيا، و النتيجة من الاختبار الميداني من المعلم هي 0/\% و من الطلاب ش.ر\% في فئة جيدة أيضا. أما نموذج التعلم المناسب لتطبيق المنتج المطور هو التعلم بأسلوب لئ لمب لئ

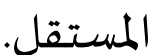

الكلمات الأسـاسية: أندرويد، نظام الإنجليزية، مهارة الكلام، الوسيلة التعليمياة.

Abstract: The purposes of this study were to produce an effortless English system instructional media using android application to improve speaking skills in Arabic, to determine the feasibility of the developed product, and to find out the appropriate learning model to implement the product. This study used Dick and Carry development model. The developed product was an application called Sabla which has several themes, namely: Family, Introduction, and My Home. Each theme has at least four components, including reading text, video, short story, and 
vocabulary. The results of product validation described the instructional expert's judgment was $80.5 \%$ in the good category and the media expert gave $86.1 \%$ in the good category too, while the field test given to teachers was $85 \%$ and to students was $83.3 \%$ in the good category too. The appropriate learning model to implement the developed product was self-learning model.

Key words: Android, effortless english, speaking proficiency, learning media.

نقل الاستنتاج لجودران أن

النجاح في تعلم اللغة الأجنبية يعتمدعلى لهودراج

مدى الاتصالات بين المعلمين والطلاب

تجرى (Chaudron, 1988: 10)، ومن ثم،

التي ينبغي مراعاتها هي التفاعل أو

التواصل بين المعلمين والتلاميذ، خاصية

في لغة الهدف. ولذلك، فإن النهج الأوضح في تعلم اللغة، وفقا لكامل الناقة، هو نهج السمعية الشفوية (الناقة، 1910)، لدياء عنصرين الأولين،

وهما الاستماع والكلام (الناقة، 1910):

ولكناه يحتاج إلى تحقيق توازن

بين تسهيل براعة الطلاب مع استخدام لغة الهدف وتوقّع الحالة النفسية

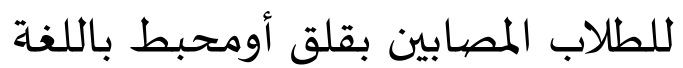

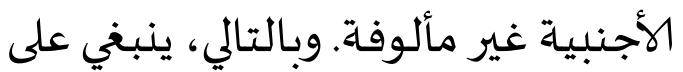
المعلم أن يرشد الطلاب لكي يكونوا قادرين على التعبير و تطوير أفكارهم فى لغةالهدف عفوية وسلاساة، وبجانب ذلك، هم يملكون الدوافع لأن يفعلوا تلك الكفاءة (Howard, 2016: 7).

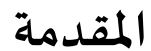

كانت في تعليم اللغة الأجنبية

مشكلة الفجوة مفتوحة على مصراعيها، وربما عن قصيد لا تغطي. فإنها تتربط

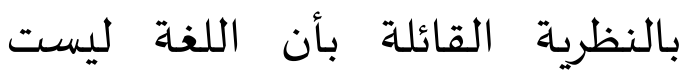
المبحث المدروس فقط، بل وسائله التعليمية أيضا (Chambless, 2012:142). في هذه الحالة، تواجاه معلم اللغة الأجنبية معضلة متعلقة بقدرته على استخدام لغة الهدف في الفصول الدراسية. والفجوة بين النظرية والتطبيق تنشأ هذا الضعف. ومن ناحية النظرية، يمكن تعلم اللغة الأجنبية تعظيما عن طريق بناء الفصل كالمرآة للعالم الحقيقي ( Gil \& Salazar 133 كوسيلة الاتصال فقط والفصل كالمظهر من مظاهر ثقافة الهدف. وبالإضافة إلى ذلك، فإن العوامل الداخلية مثل المخاوف المفرطة حول استخدام لغة الهدف عامل باهظ للمعلمين على التواصل باستخدام لغة الهدف على

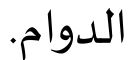


بالولايات المتحدة يسمى بـ أ.ج. هوغ. هذا

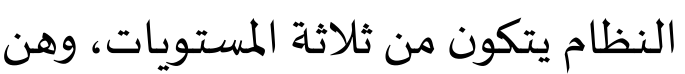
المستوى المتوسط المنخفض، والمستوى المتوسط، والمستوى المتوسط العالي. تشمل كل المستوى على الموضوعات المختلفة من الدروس، وكان في كل الموضوع حزمة من الدروس بشكل من لمدون

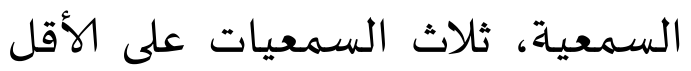

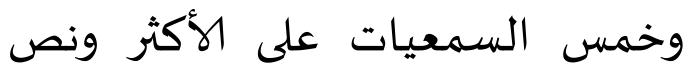
واحد بشكل ب.د.ف (PDF). الدرس الأول هو درس سمعية الذي يحتوي على

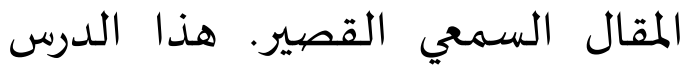

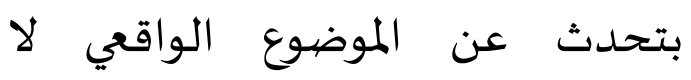
الاصطناعي. الدرس الثاني هو درس الموهي

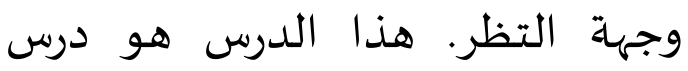
القواعد ولكنه بدون تعلم حكم القواعد، بل بممارسة استماع القصية القصيرة مع وجهة النظر المختلف. الدارس غير مطلوب بأن يفكروا عن حكم النحو أو الصرفي، بل هم يتعلمون القواعد تلقائيا. الطلاب سيتعلمون أن أن يستعملوا القواعد بلا تفكير. الدرس الدوسيع الثالث درس المفردات الذي يحتوي على العبارات والمفردات العامة وكثيرة استعمالها في التفاعل اليومي. الدرس الدرداس وهيرة الرابع يسمى بدرس القصة القصيرة،
قد واجه المشكلات التعليمية

المتساوية أ.ج. هوج (Hoge)، وهو مدرس اللغة الإنجليزية ومخترع نظام الإنجليزية بدون صعوبة (Effortless English). خبراته كالمعلم في أجزاء مختلفة من العالم أدت

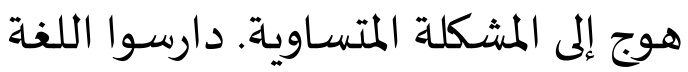
الإنجليزية مصابون بالملل والإحباط والجهاز العصبي، ومعظمهه لاتزالون يصعبون أن تحدثوا بطلاقة و كانوا دارسين منذ سنوات (Hoge, 2014: 17). وجذر هذه الحالة، وفقا لهوج، النظام والمنهج المستخدم ما زالت قليديا وهو طريقة تركيب و ترجمة. من ثم، هوج

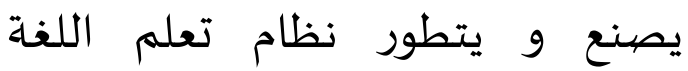
الإنجليزية المشههورب قواعده السبعة. لتنفـذهم، قدم اتصالات في اتجاه واحد، مثل مونولوج، بشكل الوسائل

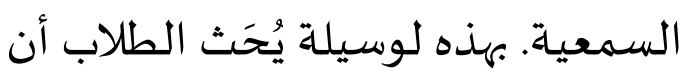
يستمعوا اللغة الثانية في شكلها الأصلي. وتعريف الأصلي هنا هو أصيل، وكاد أن

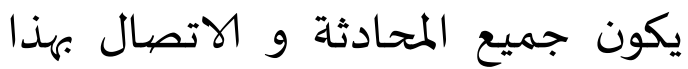
المدخل هي الطبيعية، عفوية، وبدون

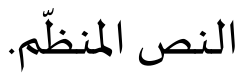
الانجليزية بدون صعوبة هي النظام الذي صنعها معلم اللغة الانجليزية من سان فرانسيسكو 
الأخطاء، الميل إلى الإهتمام باللغة لا بالمحتوى. لقد أخذت عملية الاتصال في السنوات الأخرة تزداد بين الدول، فنما حجم التجارة، وانتشرت برامج الإذاعة والتلفزيون، واتسعت المسروعات والمعونات الفنية، وكثرت عملية التبادل الثقفي والتعليمي بين الدول، مما ادى ولمات

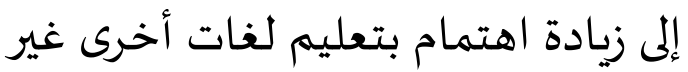

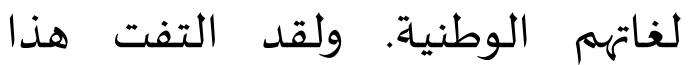
الاهتمام بتعليم لغات أخرى إلى ضرورة أن يكون الإنسان قادرا على التكلم باللغات الأجنبيات وفهمها، ومن ثم الأن انعكس ذلك على الطروق التي يتعلم بها الناس قراءة اللغة الأجنبية بطلاقة، والكتابة فيها في دقة.

الطريقة هي عبارة عن خطة

عامة لاختيار وتنظيم وعرض المادة

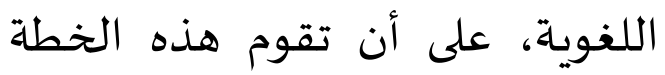
بحيث لا تتعارض مع المدخل الذي

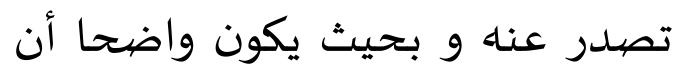
المدخل شيء مدئي والطريقة شيء بهون

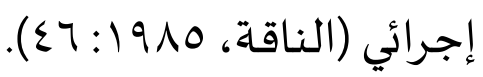

هذه الطريقة السمعية الشفهية

هي التي استخدمت أول ما استخدمت السمعيه الئقديه أول في تعليم العسكوريين الأمركيين
وهناك الائيلات البسيطة والسهلة تناسب على القصة القصيرة بعد قرائتها. الدارس مطلبون أن يجيبوا لهل

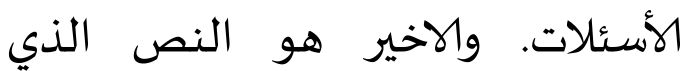
سيساعد الطلاب لأن يفهموا المقال والقصة القصيرة إذا يجيدون المشكلة.

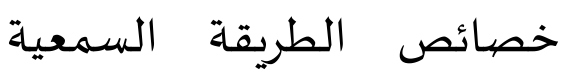
الشفهية أي الطريقة الأسساسية في نظام الإنجليزية بدون صعوبة، كما لقال دوجلاس براون فيما يلي: تقدم المادة الجديدة في شكل الحوا، يكون الإعتماد الأكبر على المحاكة، وتذكر العبارات، وزيادة التعلم، تقدم البنى عن طريق المبره التقابل، ولا تقدم إلا بنية واحدة في وقت واحد.، تعلم الأنماط البنائية

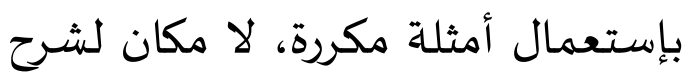

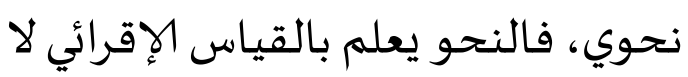
بالشرح الإستنباطي، تحدد فيدو المفردات تحديدا صارما، وتقدم في سياق، توجياه

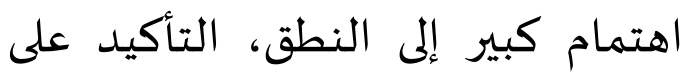

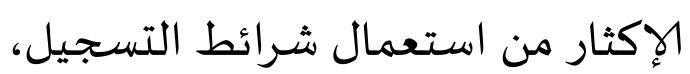

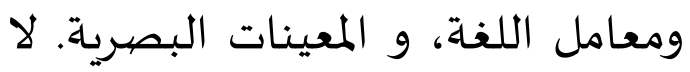

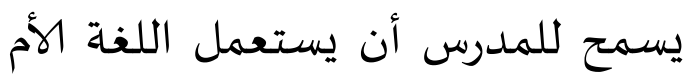

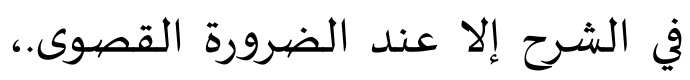

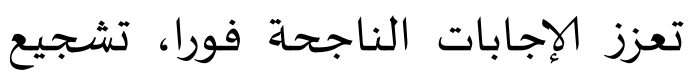

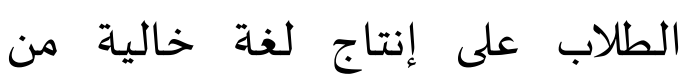


صورة بصوت أو عروض مسرحية أو تمثيلية أو عينات أو نماذج أو زيارات ميدانية أو غير ذلك من الوسائل إما سمعية أو سمعية وبصرية - كلها قادرة على إعطاء المتعلمين والمتدربين خبرات ومعارف ومعلومات تختلف في قوتها وثباتها ودقتها وتأثيرها على الشرح اللفظي المجرد من خلال اللفظية البحتة (منصهور، سم/9 1: ع).

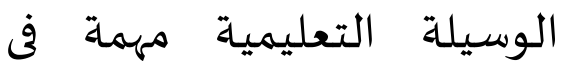
تعليم اللغة العربية الأجنبية، خاصية في الفيه تعليم اللغة العببية، لأهها تسهل المعلمون في التعليم والتعلّم. الوسيلة التعليمية هي كل أداة تستخدمها المعلم لتحسين عملية التعليم، وتوضيح

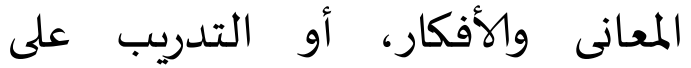
المهارات، أو تعويد التلاميذ على العادات

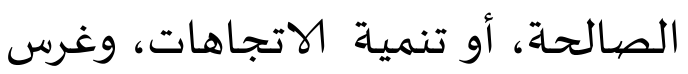

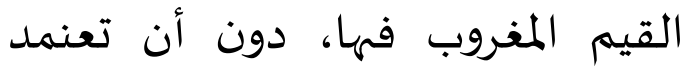
المعلم على الألفاظ والرموز والأرقام .(Sadiman, 1992: 16)

إن تكنولوجية علم من العلوم الحديثة التي تهدف إلى تسهيل عملية التعليمياة عن طريق استفادة مصادر التعليم والتعلّم. سوف تنمو التعليم عندما تقدم العلوم والتكنولوجيا.
اللغات الأجنبية لإرسالهم في مهارات خارج بلادهم بعد الحرب العالمية

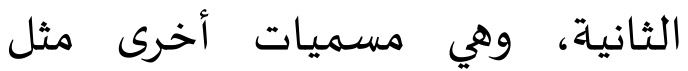

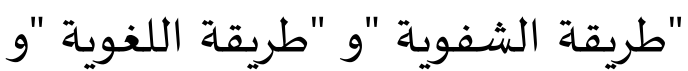
آان اسمها أول ما ظهرت لهرت لأسلوبه

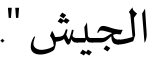

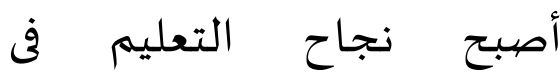

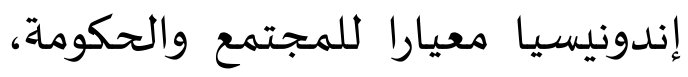
خاصة في مادة اللغة العربية، ولكن هناك تسبب عوامل: انخفاض تطبيق استراتيجية في في التعليم والتعلم والخفاض طرائق التدريس وخاصة انخفاض الوسيلة التعليمية على أساس واس إسلى

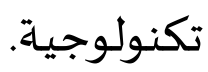

أصل كلمة الوسيلة (media) من اللغة اللاتينية وهي جمع من كلمة

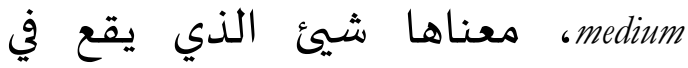
الأوسط (بين الحزبين) أو الآلة. المقصود منها الوسائل التعليمية كالوسيطة التي ترسل الرسالة التعليمية بين المرسل إلى الى الى اليهايه قابل الرسالة. عند AECT (Association For Education and Communication Technology) الوسيلة هي شيئ مستخدم لتوصيل المعلومات (Anitah, 2010:4). مما لا شك فياء أن الوسائل التعليمية - سواء كانت في صورة رموز لفظية أو صورة ثابتة أو متحركة أو 
أأقل. يمكن الطلاب والطالبات أن يدرسوا أي المواد الدراسية سواء كانوا يستطيعين لعب الوسائل الإجتماعية كفسبوك أو واتسأب أوتويتر. وبأن يجئ لونئ نمو الزمان، ينبغي أن يكون التنمية في مجال التربية، منها تطوير وسائل التعليمية الأندرويدية لكي يستطيعوا الطلاب والطالبات أن يدرسوا في أي لتعليه مكان ما ولا في المدرسة فقط. تطبيق أندرويد هو نظام تشغيل مجاني ومفتوح المصددر مبني على نواة

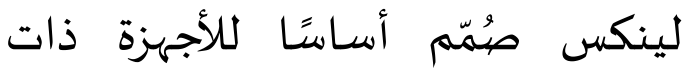
شاشات اللمس كالهواتف الذكية والحواسب اللوحية، يتم تطوير الأندرويد من قبل التحالف المفتوح للهواتف النقالة الذي تديره شركة جوجل. تستند واجهة مستخدم أندرويد

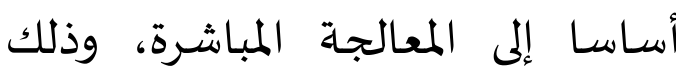
باستخدام الإيماءات اللمسية التي لمبات التهرهات تتوافق إلى حد كبير مع الحركات

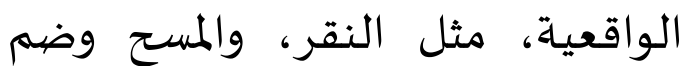
الأصابع، من أجل التعامل مع الأشياء

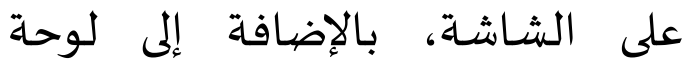
المفاتيح الافتراضية لإدخال النص. بلاهياه بناء على ذلك، الوسيلة التعليمية على أساس الأندرويد تستطيع
وكانت المصطلحات منها اتصالات (محم)

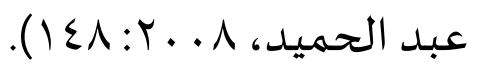
تطوير تكنولوجية تستطيع أن تستفيد في عملية اللغة العربية. تكنولوجية تتكون من مطبوعة وغير مطبوعة مثل الإلكتروني. الوسائل المطبوعة منها، جريدة ومجلات وكتب ونشرة وفـديوهات والسمعية وغيرها. لما وسائل الإلكتروني منها، حاسوب وتلفاز

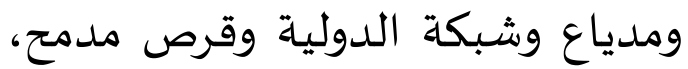
(Rasyidi, 2009: وتطبيق أندرويد وغيرها

الوسيلة الإلكتروني مهمة في عملية التعليم، مثل في المدرسة المانية المتوسطة الإسلامية الحكومية الثانية كديري. هذه المدرسة تستخدم تكنولوجية في عملية التعليم ولمعظم الصفوف، ولكن أقلية المعلمين يستخدمون الوسيلة الإلكترونية.

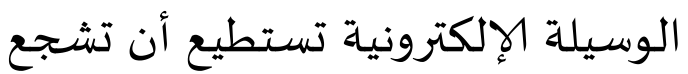

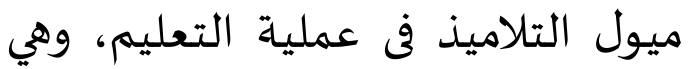
تطبيق أندرويد.

وإلى جانب ذلك، زمان الآن زمان التكنولوجية. معظم الطلاب والطالبات في مستوي المتوسطة يملكون التجهزات الإلكترونية مثل الهاتف الذاكي على 
توفره الذي يمكن أخذ المسؤولية منه .(Sukmadinata, 2009: 164)

والمدخل المستخدم في هذا

البحث هو الكيفى والكمي لأن البحث وصف بالوقت وازدهر الباحث فى ميدان

البحث (Sukmadinata, 2009: 314). المدخل الكيفى كثيرا ما يحاول أن يعطي زيادة العلوم في الوصفى تفصيليا عن الوقوع الطبيعي الذي للم يوصف كاملا في الأديب. يعطي المدخل الكيفى الإعانة

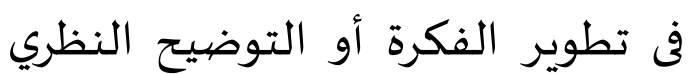
بما يلاحظ (Trianto, 2010:235). بناء على ذلك، هدف هذا البحث إلى استعمال نظام الإنجليزية بدون صعوبة ت نffortless) English)

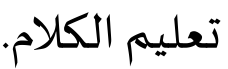

\section{نتيجة البحث ومناقشته}

في هذا المبحث سيبين الباحث

عن نتائج تحليل البيانات. سيشرح هنا

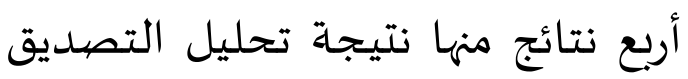

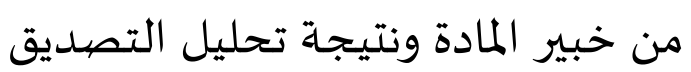

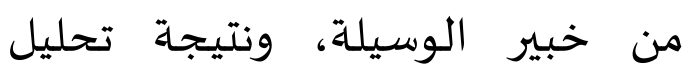

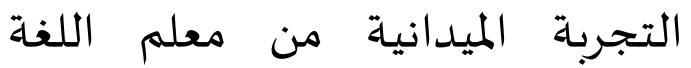
العربية، ونتيجة تحليل التجربة

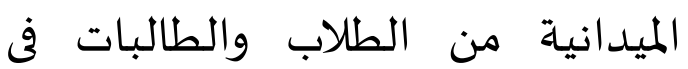

أن ترقي مهارة الكلام، لأن لديها جاذبية

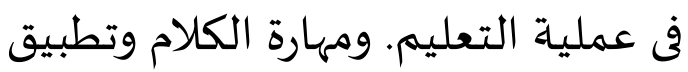

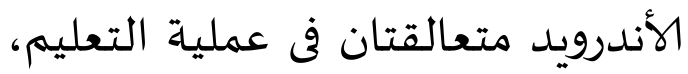
لذا فـجيب في عملية التعليم أن يوجد الندان مادة التعليمية والوسيلة التعليمية لترقية التعليم. واستنادا إلى المشاكل والظواهر التي تم وصفها، يرى الباحث أن تطوير وسيلة تعليم اللغة العربية بنظام الإنجليزية بدون صعوبة ضروري جدا. ويأمل الباحث، من هذا التطوير، بأن دارس اللغة العربية لا يشعر مخاوف

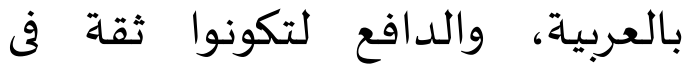
استخدامه. ويرجو الباحث، أن يسهّل

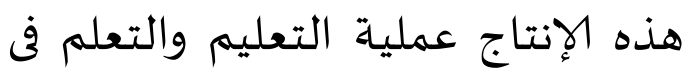
الفصل، و تحفيز المعلمين أن يستخدموا اللغة العربية للإتصال

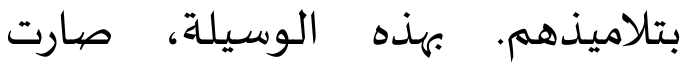
اللغةالعربية أسهل و متفاعل.

طريقة البحث استخدم الباحث طريقة البحث التطويري واستخدمه لانتاج المنتاج المعين (Sugiyono, 2010: 311). البحث و التطوير (Research \& Development) هو عملية أو خطوات يستحدم بها تطوير

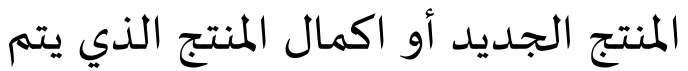




$$
\text { ب. المفردات }
$$

المستخدم يستمع هذه

المادة بلدون حفظ الكلمات.

يستمع هذه المادة مرة حتى

ثلاثمرات فى اليوم لمدة أسبوع.

ب. القصية القصيرة

لأن هذه المهادة أهم

وأقوى المواد فى هذا النظام، على هلى الماه

المستخدم أن يستمع أكثر من

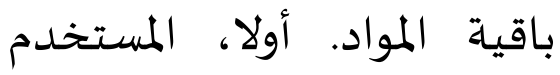

يستمعها، ثم يتوقف مؤقت بعد

كل سؤال، أجيب السؤال ثم

تشغيل من جديد. يواصل

التوقف والإجابة لكل الأسئلة.

لايفهم بعض الكلمات أوكلها أمر

عادي. فى هذه المادة السمعية

يجب على المستخدم أن يفعل ما

الآتي:

أ) سيقال التعبير، وبعده

المستخدم مطلوب أن يقول

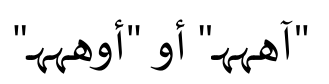

ب) سيقدَّم الأسئلة المعلومة المعلة

جواباء، وعلى المستخدم

الجواب. لا جملة المفيدة

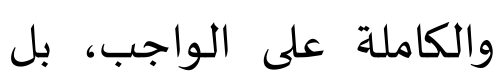

الصف السابع فى المدرسـة المتوسطة الإسالامية الحكومية الثانية كديري.

$$
\text { نموذج التعلم باستخدام المنتج }
$$

فى هذا المبحث سيأتي بيان

نموذج التعلم باستخدام المنتج. ولكناه

يحتاج أن يشرح قليلا النظاميات

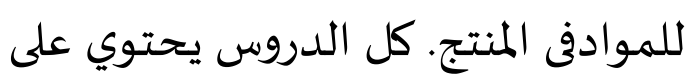

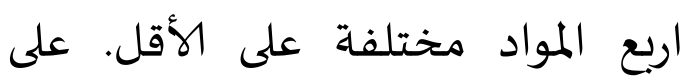
المستخدم أن يدرس الدرس أي كل المواد فيـ معا. سوف يستمع إلى جميع المواد فى درس واحد فقط لملدة أسبوع أو أكثر. لن يرقي كفاءتاه إلا اتباع المستخدم هذه الطريقة. ولكن بعض الدروس لديهم اكثر من أربع المواد الدراسية. أما شرح نموذج التعلم لكل مادة تفصيليا كما يالي:

$$
\text { أ. المواد الدراسية }
$$

يقرأ الكاتب المادة، ثم يراجع

قائمة الكلمات، ولا يُقترَح أن يدرسها ولا يحاول أن يحفظها.

يكفى بقراءتها عدة مرات كل يوم

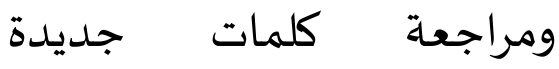

(بطريقة مريحة). ويُقترَح

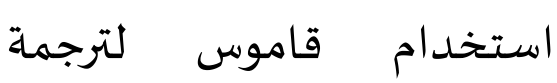

الكلمات الصعبـة، إذا كان ملزما. 


$$
\begin{aligned}
& \text { المستخدم يشاهد و } \\
& \text { يستمع و يتسمتع هذه المادة. }
\end{aligned}
$$

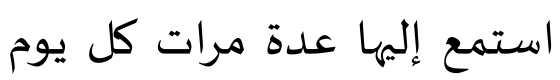

$$
\begin{aligned}
& \text { لمدة أسبوع على الأقل. الهدف مرات إلها على } \\
& \text { من هذه المادة هو فهم . . 1. } \\
& \text { حالا وتلقائيا. }
\end{aligned}
$$

0. القصة القصيرة على وجهة

$$
\text { النظر }
$$

هذه هي طريقة لترقية

طلاقة النحو والصرف، بدون

دراستاه. استمع جيدا واسترح. لا

$$
\text { تفكر القواعد النحوية. }
$$

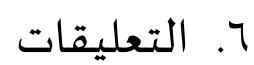

هذه

اضيافـة حول الموضيوع. قد تبدو

بشدة صعبة. إذا بدت صعبة

جدًا ، فتخطها. مادة التعليقات هي أقل أهمية المادة في نظام الإنجليزية بدون صعوبة. يمكن تخطيها إذا شعر بصعوبة. وبرغم

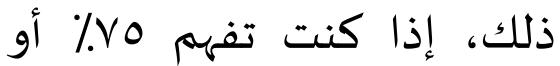
أكثر، فاستمع. ربما لن تفهم كل إدال شيء، هذا عادي. استرح واستمع فقط. ركّز على الفكرة الرئيسية والمعلومات، ولا على اللغة. حاول فهم الأفكار الرئيسية. استمع إلى ولى الى
يمكن أن يجيب بكلمة أو كلمتين فقط. ج) سيقدم الأسئلة غير المعلومة

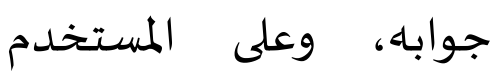
التخمين وقول التخمين بالجهر فورا. ويمكن التخمين

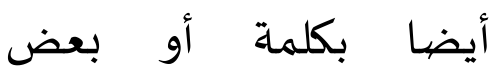
الكلمات فقط. لا جملة المفـدة والكاملة على لهملى الواجب.

عندما تجيب على سؤال، لا تقلق بشأن الإجابات الموافقة. قول إجابة صحيحة وموافقة ليس ههم. الأهم هو السرعة. حاول أن يجيب بشدة سرعة

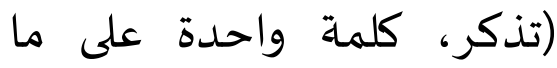
يرام). السرعة هي الهدف. تطلَب الإجابات السريعة والقصيرة، ولاإجابات طويلة.فى البداية، قد

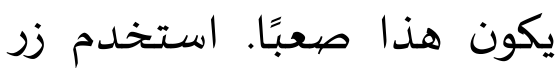
التوقف. حاول، في الهاية، الإجابة فورا، دون استخدام زر التوقف. قد يستغرق هذا الأمر أسبوعين أو أكثر. ع. الفيديو 
وتلقائية. أفضل جدول الدراسـة هو

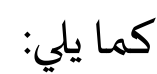

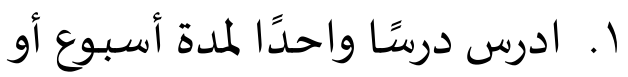
أكثر. هذا صحيح، درسًا واحدًا

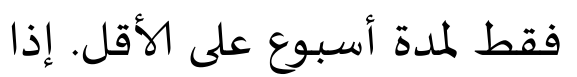

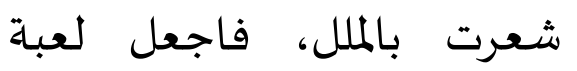
بمادة القصية القصيرة. حاول

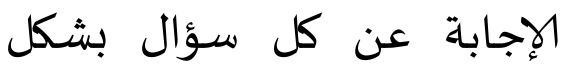

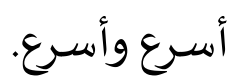
r. كل يوم، استمع إلى كل الموادفى الدرس. حاول الاستماع إلى كل إلى لمون مادة (فى الدرس) مرتين على الأحة الى الأقل كل يوم. استمع إلها كل المان يوملمدة أسبوع على الأقل. لذا لذا لها r. يجب عليك تكرار كل مادة

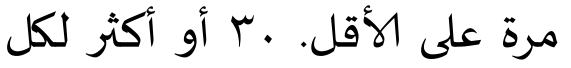

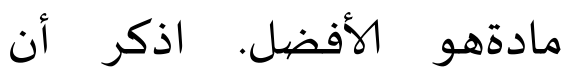
القصية الصغيرة وقصةالصغيرة على وجهة النظر هما الأهم. r. استمع على الأقل ساعة واحدة كل يوم. ساعتان كل يوم هي الأفضل. يمكن أن تقسّم وقتكفـاليوم. على سبيل المثال، يمكنك الاستماع لمدة .ب دقيقة سئل في الصباح، و ·r دقيقة عند

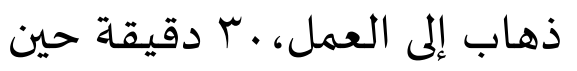

هذا الدرس عدة مرات كل يوم لمدة أسبوع على الأقل.

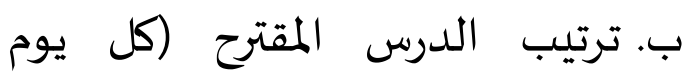
باستخدام الدرس المتساوي): 1. اقرأ مادة نص القراءة. اقرأ وراجع ذلك، لا "تدرس" ذلك. لا تحاول أن تحفظها. r. استمع إلى مادة المفردات عدة أنهابا مرات

r. استمع إلى مادة القصة القصيرة عدة مرات ع. استمع إلى مادة القصة القصيرة على وجهة النظر، إن كاده إنت الن الن

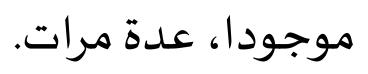

0. شـاهد واستمع إلى الفـديو عدة مردة

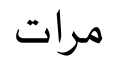

7 ا. استمع إلى التعليقات مرة واحدة

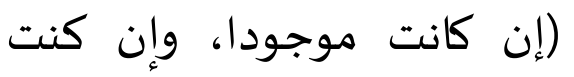
تستطيح فهمها). ج. جدول التعلم الإنجليزية بدون صعوبة هي نظام التعلم العميق. سوف تتعلم كل المادة بشـدة جيد و دقيق. سوف تسترخي وتدرس بالبطء وتعلم ان تتكلم اللغة العربية بسهولة ولدية 
إذا بدت الدروس سهلة، حاول

الإجابة عن الأسئلة بشدة سرعة، إدالة اجعلها لعب.

إذا تبدو دروس في مستوى لعاب

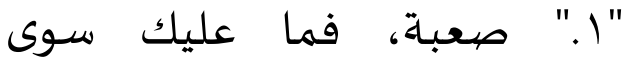

الاسترحاء. يمكنك الاستماع إلى درس

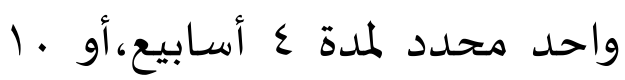

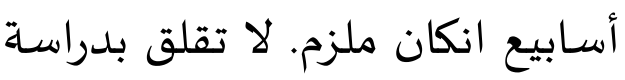

كثير من الدروس. هذا ليس مهما.

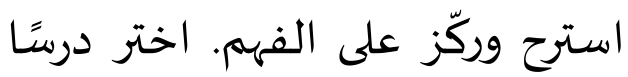

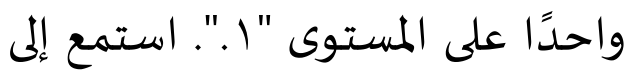

جميع الموادفهكل يوم حتى تفهمهيم

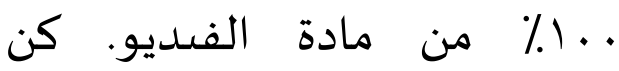

بالباطء. سوف ترقيلغتك كثيرا، ولو

كنت تستمع إلى درس واحد فقط

لمدة • ا أسابيع(A.J. Hoge, 2008).

بناء على العرض ولمات ومناقشة

البيانات السابقة يقدّم الباحث النتائج

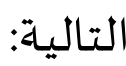

أ. تصيميم الوسيلة التعليمية بنظام

الإنجليزية بدون صعوبة باستخدام

تطبيق أندرويد لترقية مهارة الكلام

حصل الباحث على تطبيق أندرويد

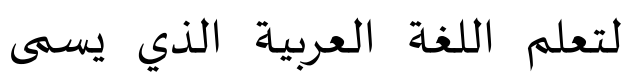

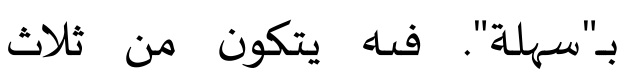

الدروس منها "الأسرة"، و"التعرف"، منكون
الغداء ، و • r دقيقة عند رجوع

إلى المنزل، و.r دقيقة قبالالنوم.

فى الواقع، هذاهي طريقة ممتازة

للتعلم.

ع. استرح ولا تفكر القواعد

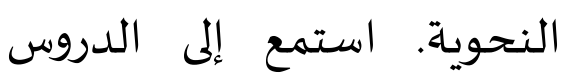

واستمتع بها. لا يوجدالضغط النحونه

هنا. ما عليك سوى الاستماع

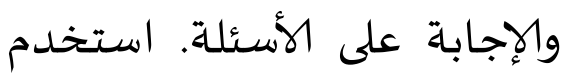

زر التوقف. إذا كنت تواجه

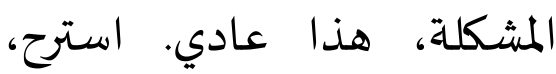

واعد إلى البداية، واستمع أكثر.

الاستماع هو أهم الأنشطة.

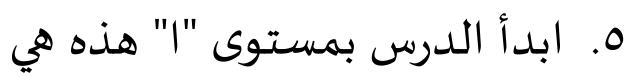

أبسط وأقصر وأسسل الدروس.

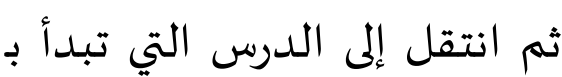

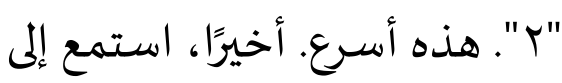

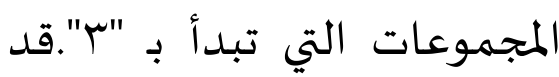

يكون ذلك صعبا، اعتمادا على

مستواك.

رغم أن يبدو الدرس سهلة،

استمع إلى درسا واحدا فقط لمدة

أسبوع كامل. عرفتَ الكلمات حسنا

من قبل، لكنك تحتاجهم أن

يتعمقوا في دماغك. تحتاج إلى كثير

من التكرار وكثير من إجابةالأسئلة. 
اللغة العربية في الصف الأول عن استخدام الوسيلة التعليمية على أسـاس تكنولوحبا خاصية في

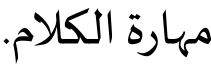

Y. تصميم المنتج، إجابة عن عن الحاجات والمشكلات المطروحة بيانها في السبق، جمع وصهمم الباحث الأشياء المحتاجة قبل جهيل وهيل تطوير الوسيلة التعليمية بتقديم وإعداد والبيانات. المنهج الدراسي المادة اللغة العربية خاصة في تعليم

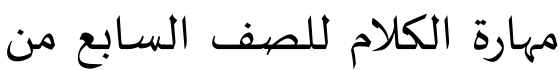

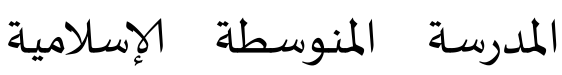
الحكومية الثانية كديري. r. تطوير المنتج، في تطوير الوسيلة

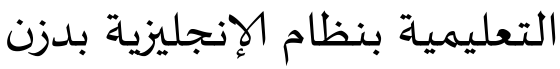

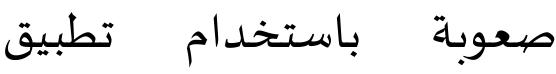
أندرويد لترقية مهارة الكلام، بهدية

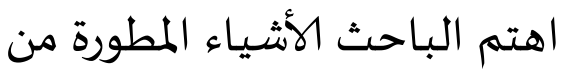
الكتاب التعليمي على وزارة الئؤون الدينية خاص فى المادة مهارة الكلام الذي نقص الوسيلة

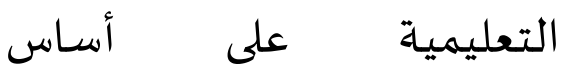
تكنولوجبا، وكذلك باستخدام

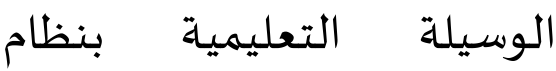

و"بيتي". كل منهم تتكون من اربع

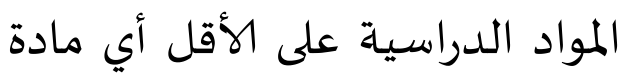
نص القراءة ومادة فـديو ومادة القصة القصرة ومادة المفردات. وهو مطور من كتاب التعليمي وزارة الشؤون الدينية. وهذه الوسيلة التعليمية على أساس تكنولون وتتكون من السمعية وفـديو والصور وألوان المنتوعة. وهي من الوسائل السمعية البصرية. وأما تصميم التطوير الذي استخدماء الباحث لتطوير الوسيلة التعليمية بنظام الإنجليزية بدزن صعوبرة

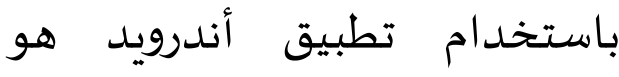
باستخدام منهج البحث التطويري

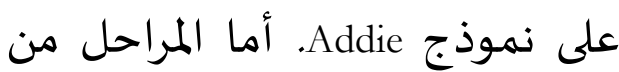

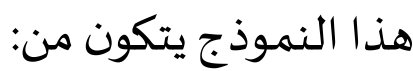
1. تحليل الإحتياجات، حلل الباحث

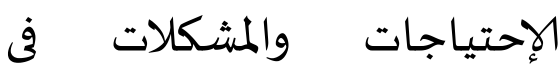
المدرسة المتوسطة الإسلامية الحكومية الثانية كديري. تحليل الإحتياجات بمقابلة مع رئيس

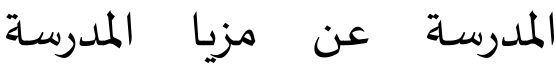

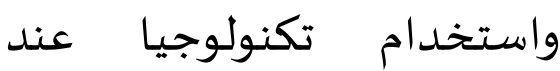

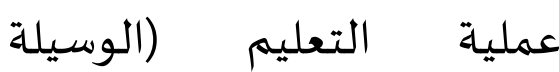
التعليمية) ومقابلة مع المعلمة المعلم 
والطالبات في صف السابع تعرض

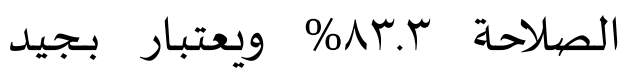

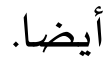

ج. أما تعليم مهارة الكلام باستخدام الوسيلة التعليمية بنظام الإنجليزية

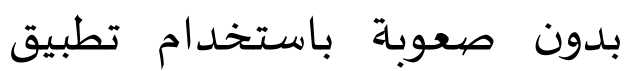

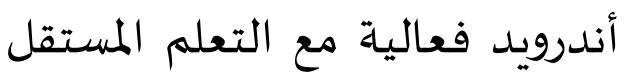

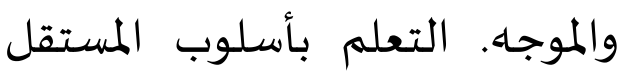
والموجاء يعني أن الطلاب والطالبات يمكن تشغيل أجهزة الهاتف الذكي بمفردهم حتى يتمكن الطلاب من

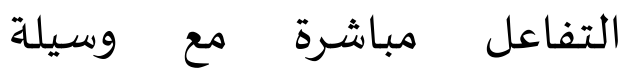
التعليمياة، ويكون المعلم مشرفا في

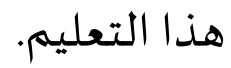

\section{الخلاصية}

بالنسبة للمطورين، يمكنهم تطوير وسيلة تعليمية أكثر تنوعًا وابتكارًا. متغير من حيث الدروس وعرض الوسائط والتمارين والرسوم مئرئ

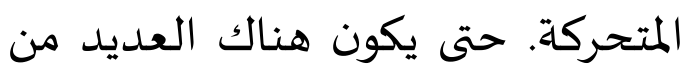
وسائل التعليمية التفاعلية التي تم تطويرها لتحسين التعلم، وخاصة تعلم اللغة العربية. مع وجود وسيلة التعليمياة بأكثر تنوعا، فمن المتوقع، أن وجناء تحسّن نوعية التعليم في إندونيسيا.
الإنجليزية بدزن صعوبة

باستخدام تطبيق أندرويد (تكنولوجيا) التلاميذ يستطيع أن يرغبوا في تعلم اللغة العربية خاصية فى مهارة الكلام. ع. في تجربة المنتج من الويبلة التعليمية،، قام الباحث باجراء

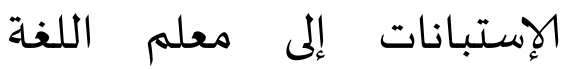
العربية والطلاب والطالبات في المبات صف السابع أ من المدرسة ولهابة المتوسطة الإسلامية الحكومية الثانية كديري. ه. المرحلة الأخرة هي تقويم المنتج إلى خبيرين هما خبير المادة وخبير الوسيلة وإلى الطلاب والطالبات في صف السابع أ من المدرسة المالية المتوسطة الإسلامية الحكومية الثانية كديري. ب. نتائج التصديق والتجربة الميدانية كما يليي: التصديق من خبير المادة

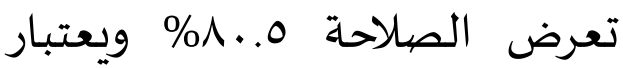
بجيد. وأما التصديق من خبير

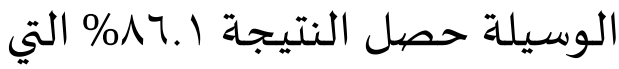
تعتبار بجيد أيضيا. كانت تجربة التهيلة الميدانية من المعلم حصلت درجة

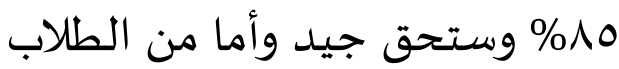


المملكة العربية السعودية:

جامعة الامام محمل بن سعود

$$
\text { الإسلامية. }
$$

منصور، عبد المجيد سيد أحمد. rی19 ا. سيكولوجية الوسائل

التعليمية و وسائل تدرلس

اللغة العببية. الطبعة الأولى.

$$
\text { القاهرة: دار المعارف. }
$$

الناقة، محمد كامل. 1910. تعليم اللغنة

العبية للناطقين بلغات أخرى:

أسسيه-ملدخله-طرق تلدربسه.

المملكة العربية السعودياة:

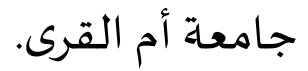

Anitah, Sri. 2010. Media Pembelajaran. Surakarta: Yuma Pustaka.

Arikunto, Suharsimi. 1995. Dasar-Dasar Evaluasi Pendidikan. Jakarta: Bumi Aksara.

Arikunto, Suharsimi. 2006. Prosedur Penelitian: Suatu Pendekatan Praktik (Edisi Revisi VI). Jakarta: PT Rineka Cipta.

Chambless, K. 2012. Teachers' Oral Proficiency in the Target Language Learning: Reasearch on Its Role in Language Teaching and Learning. Foreign Language Annals, 45 (1), 141162.

Chaudron, C. 1998. Second Language Classroom: Research on Teaching and Learning. Cambride: Cambridge University Press.
براون، دوجلاس. \&199. أسس تعلم اللغة و تعليمها. بيروت: دار النهضية العربية.

حكمت، الحلو. 9 . . . . مشكلات الطفل السسلوآية في البيت و الممدرسة. القاهرة: دار النشر للجامعة.

الحميد، محم عبد. رم..ب. الهجوم. مالانج: جامعة مولانا مالك إبراهيم الإسلامية الحكومية. الخولي، محمد علي. 1917. أساليب تلدريس اللغة العببية. الرياض: المملكة العربية السعودية.

سلطان، محمود السيد. .19V. دراسات في التوبية و المجتمع. القاهرة: دار المعارف.

طعيماة، رشدى أحمد. ع . . r. المهارات اللغوية مستوياتها تدريسها

صعوباتها. القاهرة: دار الفكر<smiles>[AlH2]=[AlH2]</smiles>

العصيلي، عبد العزيز بن ابراهيم. r r. r. . طرائق التدريس اللغة العببية للناطقين بلغات أخرى. 
Rosyidi, A. W. 2009. Media Pembelajaran Bahasa Arab. Malang: UINMalang Press.

Sadiman, A. 1992. Media Pendidikan, Pengertian, dan Pengembangan. Jakarta: PT Raja Grafindo.

Setiyadi, A. B. 2006. Metode Penelitian untuk Pengajaran Babasa Asing: Pendekatan Kuantitatif dan Kualitatif. Yogyakarta: Graha Ilmu.

Sugiyono. 2010. Metode Penelitian Pendidikan Pendekatan Kuantitatif, Kualitatif dan R\&D. Bandung: Alfabeta.

Suja'i. 2008. Inovasi Pembelajaran Bahasa Arab. Semarang: Walisongo Press.

Sukmadinata, N. S. 2009. Metode Penelitian Pendidikan. Bandung, PT Remaja Rosdakarya.

Tim Puslitjano. 2008. Metode Penelitian Pengembangan. Jakarta: Pusat Penelitian Kebijakan dan Inovasi Penidikan Badan Penelitian dan Pengembangan Departemen Pendidikan Nasional.

Trianto. 2010. Pengantar Penelitian Pendidikan bagi Pengembangan Profesi Pendidikan \& Tenaga Kependidikan. Jakarta: Kencana.
Gil, M. G., Garau, M. J., Noguera, J. S. 2012. A case study exploring oral languge choice between the target languge and the $11 \mathrm{~s}$ in mainstream CLIL and EFL secondary education. Revista de Linguistica y Lenguas Aplicadas, 7 , 133-146.

Hamid, A., Baharudin, U., \& Mustofa, B. 2008. Pembelajaran Babasa Arab. Malang: UIN-Malang Press.

Hoge, A. 2009. How to Use Our English Lesson. Diakses dari www.effortlessenglishclub.com. pada 27 Agustus 2018.

Hoge, A. J. 2014. Effortless English: Learn to Speak English like a Native. Nevada: Efforless Engliss LLC.

Howard, K. 2016. Building linguistic confidence: A critical study of teachers' Target Language use in the MFL classroom and its implications for students' willingness to communicate. Research \& Development Project. Submitted for the MSc Learning \& Teaching.

bttps://en.wikipedia.org Android (operating system), diakses pada 9 Februari 2019.

Putra, N. Research \& Development: Penelitian dan Pengembangan: Suatu Pengantar. Depok: PT. Raja Grafindo Persada. 